\title{
Secondary Syphilis With Hepatitis and Nephrotic Syndrome: A Rare Concurrence
}

\author{
Jasbir Makker ${ }^{\text {a, b }}$, Bharat Bajantria, c, Suresh Kumar Nayudu ${ }^{\text {a, b }}$
}

\begin{abstract}
Syphilis, a chronic multisystem disease, is caused by a spirochete, Treponema pallidum. Clinical presentation may expand to several stages including primary, secondary and latent syphilis, which may present as early or late syphilis. Nephrotic syndrome and acute hepatitis are well-known complications of secondary syphilis. To the best of our knowledge, secondary syphilis with coexisting renal and hepatic complications has rarely been reported. Here we present a rare case of concurrent nephrotic syndrome and acute hepatitis in a patient with secondary syphilis.
\end{abstract}

Keywords: Syphilis; Secondary syphilis; Hepatitis; Nephrotic syndrome; Glomerulonephritis

\section{Introduction}

Syphilis, a chronic infectious disease, is caused by spirochete Treponema pallidum. Clinical presentation may vary from asymptomatic to systemic disease with multi-organ involvement. The clinical spectrum of syphilis may be primary, secondary, latent, tertiary or congenital based on time and nature of clinical presentation. Secondary syphilis is a systemic disease characterized by maculopapular rash, lymphadenopathy with liver and kidney involvement. Though various forms of hepatitis have been described frequently in literature [1-34], concurrent presentation of both hepatic and renal complications has been rarely described [8, 14, 23, 28, 31, 34]. We present a case of secondary syphilis with features of both hepatitis and nephrotic syndrome.

Manuscript accepted for publication May 17, 2016

aDepartment of Medicine, Bronx Lebanon Hospital Center, Affiliated to Icahn School of Medicine, Bronx, NY, USA

bivision of Gastroenterology, Bronx Lebanon Hospital Center, Affiliated to Icahn School of Medicine, Bronx, NY, USA

${ }^{\mathrm{c} C}$ Corresponding Author: Bharat Bajantri, Department of Medicine, Bronx Lebanon Hospital Center, 1650 Grand Concourse, Bronx, NY 10457, USA. Email: bbanjant@bronxleb.org

doi: http://dx.doi.org/10.14740/jocmr2595w

\section{Case Report}

A 51-year-old man presented to emergency room with abdominal pain of 3-week duration and intermittent episodes of bright red blood per rectum for 1 week. He had been in his usual state of health until 3 weeks ago. He described his abdominal pain as dull aching in the epigastric and peri-umbilical regions brought upon by eating. He was also troubled by multiple episodes of non-bilious vomiting over last $3-4$ days. He was prescribed famotidine by his primary care physician, which did not alleviate his symptoms. He had episodes of rectal bleeding in the past with spontaneous resolution. However, more recently, he has been noticing few streaks of blood mixed with stool especially when he strains to move his bowels. He reported constipation with bowel movement every 3 - 4 days. He denied chest pain, palpitation, shortness of breath, diarrhea, urinary symptoms, fever, loss of appetite or loss of weight.

His medical co-morbidities included diabetes mellitus and osteoarthritis. His current medications included ibuprofen for knee pain, famotidine and metformin. He was using tobacco and alcohol dependent in the past but he had quit more than 15 years ago. He admitted frequent use of recreational drugs like phencyclidine and cannabinoids. He lived by himself and denied any significant family medical history.

On initial evaluation, he was not in distress, afebrile with temperature $98.4^{\circ} \mathrm{F}$, pulse 88 beats $/ \mathrm{min}$, blood pressure $130 / 78 \mathrm{~mm} \mathrm{Hg}$, respiratory rate $16 /$ min and body mass index (BMI) $27 \mathrm{~kg} / \mathrm{m}^{2}$. Many copper colored papules and macules were seen on the body including palms and soles of the patient (Fig. 1a, b). He was also noticed to have mild bilateral pitting pedal edema. Rest of his systemic examination including respiratory, cardiac, gastrointestinal and neurological exam was unremarkable.

Laboratory workup during his hospitalization is shown in Table 1. Serological markers of viral hepatitis including hepatitis A, B and C were negative. Autoimmune and metabolic workup for liver disease including anti-nuclear antibody (ANA), smooth muscle antibody (SMA), anti-mitochondrial antibody (AMA), liver kidney microsomal (LKM) antibody, and anti-neutrophil cytoplasmic antibody (ANCA) was tested negative. Metabolic profiles including iron studies, ceruloplasmin and celiac panel were normal. Urinalysis was remarkable with 24 -h protein level elevated to $5,915 \mathrm{mg}$ and micro- 


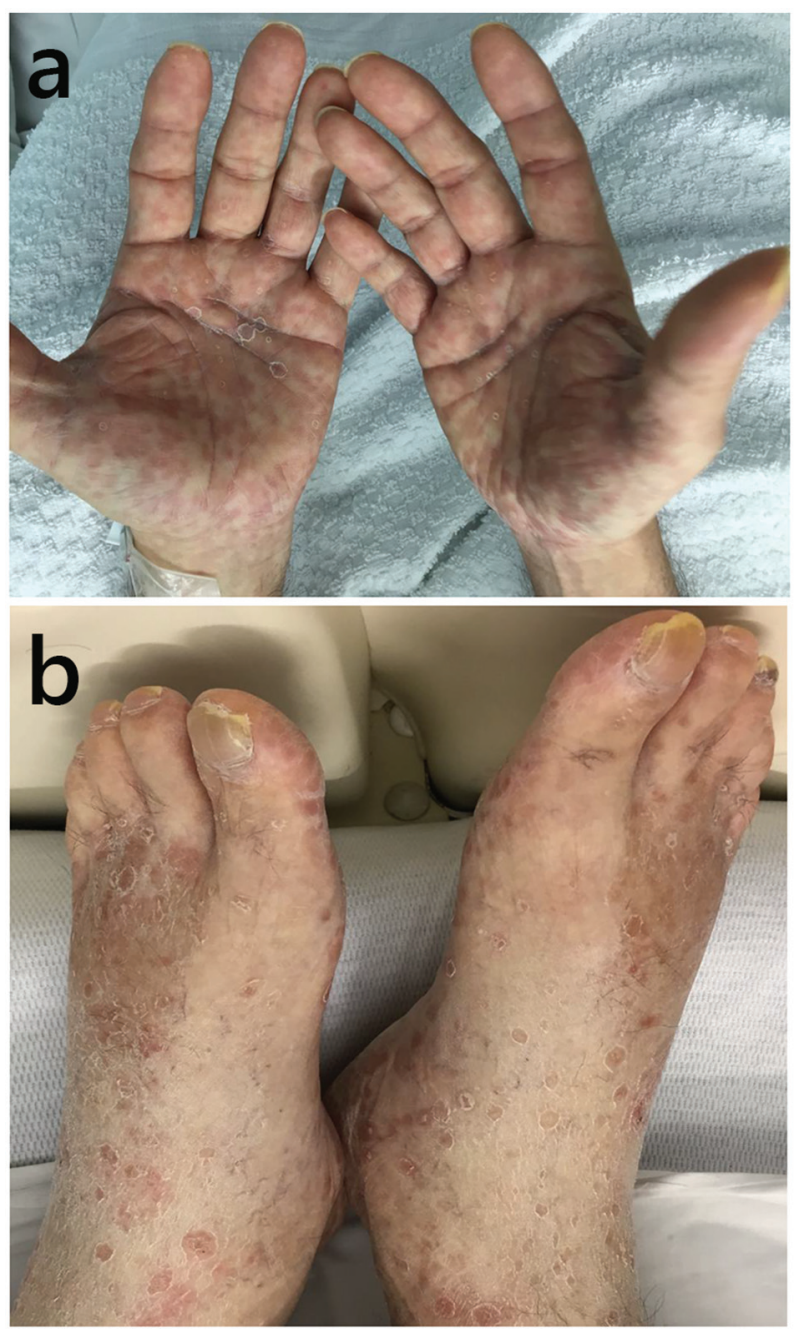

Figure 1. (a) Hands showing maculo-papular rash. (b) Feet showing maculo-papular rash.

albumin to creatinine ratio of $5,250 \mathrm{mg} / \mathrm{g}$. His chest X-ray was unremarkable. Transthoracic echocardiogram showed grade II diastolic dysfunction with normal ejection fraction of $60 \%$. Ultrasound abdomen showed normal liver, spleen, and bilateral kidneys with normal echogenicity and size.

Liver biopsy was performed and histopathology examination (Fig. 2a, b) showed chronic hepatitis with mild activity (grade 2 of 4), portal and peri-portal fibrosis with no fibrous septa (stage 1 of 4). Kidney biopsy showed segmental to global glomerular capillary wall staining for immunoglobulin $\mathrm{G}$ (2-3+), complement C3 (2-3+), complement C1 (1+), kappa $(2-3+)$, and lambda (2-3+). Electron microscopy revealed segmental to global subepithelial electron dense deposits and a focus suspicious for a cellular crescent. These findings were diagnostic of an immune complex-mediated glomerulonephritis, most suggestive of membranous glomerulonephritis (MGN). Taken together, diagnosis of secondary syphilis with luetic hepatitis and secondary membranous glomerulonephritis was made. Patient was treated with a single dose of benzathine penicillin G 2.4 million units intramuscularly.
Table 1. Laboratory Parameters

\begin{tabular}{ll}
\hline Parameter & Value \\
\hline Hemoglobin & $12.7 \mathrm{~g} / \mathrm{dL}$ \\
Hematocrit & $40.8 \%$ \\
White count & $9,400 / \mu \mathrm{L}$ \\
Platelet & $257,000 / \mu \mathrm{L}$ \\
Sodium & $125 \mathrm{mEq} / \mathrm{L}$ \\
Potassium & $4.20 \mathrm{mEq} / \mathrm{L}$ \\
Chloride & $94 \mathrm{mEq} / \mathrm{L}$ \\
Bicarbonate & $21 \mathrm{mEq} / \mathrm{L}$ \\
Glucose & $280 \mathrm{mg} / \mathrm{dL}$ \\
BUN & $19 \mathrm{mg} / \mathrm{dL}$ \\
Creatinine & $0.7 \mathrm{mg} / \mathrm{dL}$ \\
Cholesterol & $263 \mathrm{mg} / \mathrm{dL}$ \\
LDL & $205 \mathrm{mg} / \mathrm{dL}$ \\
HDL & $12 \mathrm{mg} / \mathrm{dL}$ \\
Triglyceride & $231 \mathrm{mg} / \mathrm{dL}$ \\
INR & 1.0 \\
S. protein & $7.5 \mathrm{~g} / \mathrm{dL}$ \\
S. albumin & $3.7 \mathrm{~g} / \mathrm{dL}$ \\
AST & $50 \mathrm{Unit} / \mathrm{L}$ \\
ALT & $91 \mathrm{Unit} / \mathrm{L}$ \\
Alkaline phosphatase & $274 \mathrm{Unit} / \mathrm{L}$ \\
Bilirubin (total) & $0.3 \mathrm{mg} / \mathrm{dL}$ \\
Bilirubin (direct) & $0.1 \mathrm{mg} / \mathrm{dL}$ \\
Lipase & $43 \mathrm{Unit} / \mathrm{L}$ \\
Amylase & $31 \mathrm{Unit} / \mathrm{L}$ \\
RPR titer & $1: 1,024$ \\
\hline
\end{tabular}

\section{Discussion}

The term syphilis was first coined by Hieronymus Fracastorius in 1530 but its association with Treponema pallidum was not shown until 1905, when Schaudinn and Hoffman demonstrated them in a smear from syphilitic lesion secretions [35]. In late 1940s after World War II, with the advent of penicillin, cases of syphilis in United States declined tremendously. Since then syphilis cases in United States had several peaks and falls with the all-time lowest in the year 2000 [36]. However, since the year 2000, new syphilis cases in United States have been consistently on a rise mainly among men who have sex with men (MSM) [37].

Mode of transmission is primarily through sexual contact and rarely congenital in which case vertical transmission occurs from an infected mother to the child. Syphilis can affect most organs and tissues of the human body and have varied presentations, hence earning the name "the great imitator". Syphilis, if left untreated, can progress to different stages, namely, primary, secondary, latent and tertiary. Primary syphilis starts as 


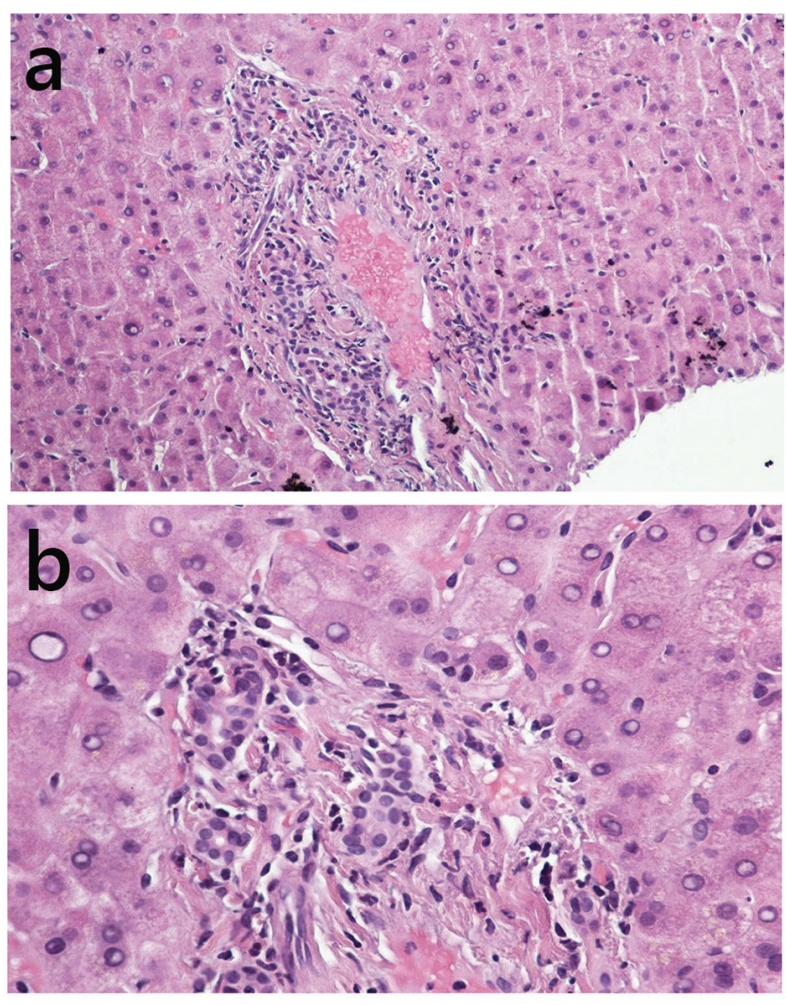

Figure 2. (a) Liver biopsy showing mild portal inflammation (low magnification hematoxylin and eosin stain). (b) Liver biopsy showing numerous polymorphs around bile-ductules (high magnification hematoxylin and eosin stain).

a painless indurated ulcer, known as chancre, mostly involving the genitalia. Secondary syphilis has a predominant maculopapular rash, lymphadenopathy with other less common manifestations like meningitis, hepatitis and nephrotic syndrome. Latent syphilis as the name suggests is an asymptomatic stage and the infected individual is diagnosed based on the positive serology. Tertiary syphilis can be cardiovascular in the form of aortitis, or neurosyphilis that may present as meningitis, vasculitis, paresis, tabes dorsalis and gumma formation [35].

Simultaneous involvement of liver and kidney in secondary syphilis is very rare $[8,14,23,28,31,34]$. Albuminuria is the most common renal manifestation in secondary syphilis. Clinically, it has a wide range of presentation from transient asymptomatic albuminuria to symptomatic nephritic or nephrotic syndrome. Pathogenesis involves direct damage by spirochetes as well as autoantibodies and immune complexes. Membranous nephropathy is the most common etiology for nephrotic syndrome in secondary syphilis. Other findings in syphilitic renal disease include mesangial proliferative glomerulonephritis, post-infectious endocapillary proliferative glomerulonephritis, rapidly progressive glomerulonephritis with crescents, minimal change disease, renal gumma and amyloid renal disease [38].

Literature on syphilitic hepatitis, also known as luetic hepatitis, has been very sparse. Most of the patients are asymptomatic but in very rare instances present with right upper quadrant abdominal pain [4]. It is now recognized that in syphilitic hepatitis, there is an elevation of transaminases with or without cholestasis. Liver biopsy shows non-specific periportal inflammation and necrosis with or without spirochetes. Liver injury occurs as a result of direct damage by spirochetes as well as via autoimmune antibodies and immune complex mediated mechanisms [39, 40].

Other gastrointestinal manifestations of syphilis are gastropathy and proctitis which may also coexist with hepatitis [6]. Gastropathy can appear like peptic ulcer disease but often has poor response to the usual treatments of peptic ulcer disease. Histologically shallow superficial ulcerations, dense mucosal monocytic and plasma cell infiltration, edema, characteristic proliferative endarteritis and panphlebitis are the most frequent findings. Syphilitic proctitis involves anal and rectal lesions that occur from anal intercourse, especially among homosexual men. Clinically, it may mimic hemorrhoids but proctoscopy shows an irregular, indurated lesion with heapedup margins and multiple small ulcerations. Biopsy specimen shows a mononuclear cell infiltrate, vasculitis and in some cases spirochetes [6].

Diagnostic methods have evolved over several years, but attempts to culture the organism have been unsuccessful. Dark field microscopy remains the quickest and most useful method to identify the organism. However, it has high false negative rates. Serological testing is the most frequently used method of testing for secondary, latent and tertiary syphilis. Serological tests are divided into two categories - non-treponemal and treponemal tests. Non-treponemal tests include Venereal Disease Research Laboratory (VDRL) and rapid plasma reagin (RPR) test that detect antibodies against cardiolipin. Treponemal tests, fluorescent treponemal antibody absorption (FTA-ABS) and micro-hemagglutination assay for Treponema pallidum (MHA-TP) antibodies have higher sensitivity and specificity as compared to non-treponemal tests [41].

Penicillin $G$ is the drug of choice due to its sustained therapeutic troughs of penicillin $\mathrm{G}$ and high susceptibility of Treponema pallidum. Single intramuscular injection of 2.4 million units of benzathine penicillin $\mathrm{G}$ is the preferred treatment for primary, secondary and early latent syphilis. Ceftriaxone has been used as an alternative by some researchers. Other drugs used to treat Treponema pallidum, especially in patients allergic to penicillin, are tetracycline or erythromycin. Treatment of neurosyphilis requires 10 - 14 days of therapy with aqueous crystalline penicillin $\mathrm{G}$ or procaine penicillin with probenecid [42]. Jarisch-Herxheimer reaction is an immunologically mediated reaction in response to syphilis treatment. It comprises constitutional symptoms like fever, myalgia, headache and worsening of cutaneous lesions [43]. There were also reports confirming resolution of hepatitis and renal complications with penicillin $\mathrm{G}$ therapy.

Syphilis is a sexually transmitted systemic disease with a broad spectrum of manifestations involving various organs at different stages of the disease. Liver and renal involvements in secondary syphilis are well-known complications. However, coexistence of liver and renal disease in secondary syphilis is very rare. Our case chronicles this rare co-existence and stresses on various presentations of syphilis as we see resurge in the incidence of syphilis and enable timely recognition with appropriate management. 


\section{Conflicts of Interest}

Authors have no conflicts of interest to disclose.

\section{Author Contributions}

Jasbir Makker and Bharat Bajantri wrote the manuscript. Suresh Nayudu reviewed the manuscript.

\section{References}

1. Rinascente C, Candela G, Cervero M, Lobato A, Carbonell A. [Acute non cholestatic hepatitis as the first manifestation of secondary syphilis]. Rev Gastroenterol Peru. 2015;35(3):247-249.

2. Saxon CJ, Helbert MR, Komolafe AJ, Higgins SP. Rash and hepatitis within days of starting a new antiretroviral regimen: nevirapine hypersensitivity, secondary syphilis or both? Int J STD AIDS. 2014;25(3):228-230.

3. Prieto-de-Paula JM, Cepedello-Perez S, Martin-Luquero Ibanez M, Franco-Hidalgo S, Uzcategui-Urdaneta MG. [Hepatitis due to secondary syphilis]. Gastroenterol Hepatol. 2014;37(9):546-549.

4. Fielding CM, Angulo P. Right upper-quadrant pain in a patient with drug abuse, secondary syphilis and occult hepatitis B virus. Med Princ Pract. 2014;23(5):471-474.

5. Ferreira-Gonzalez L, Cainzos-Romero T, FernandezFernandez FJ, Sesma P. [Secondary syphilis presenting as cholestatic hepatitis]. Enferm Infecc Microbiol Clin. 2013;31(7):487-488.

6. Adachi E, Koibuchi T, Okame M, Sato H, Imai K, Shimizu $\mathrm{S}$, Tsurita G, et al. Case of secondary syphilis presenting with unusual complications: syphilitic proctitis, gastritis, and hepatitis. J Clin Microbiol. 2011;49(12):4394-4396.

7. Kim GH, Kim BU, Lee JH, Choi YH, Chae HB, Park SM, Youn SJ, et al. Cholestatic hepatitis and thrombocytosis in a secondary syphilis patient. J Korean Med Sci. 2010;25(11):1661-1664.

8. Tsai YC, Chen LI, Chen HC. Simultaneous acute nephrosis and hepatitis in secondary syphilis. Clin Nephrol. 2008;70(6):532-536.

9. Tanyel E, Tasdelen Fisgin N, Sarikaya Genc H, Tulek N. [A case of secondary syphilis with hepatitis]. Mikrobiyol Bul. 2007;41(2):291-296.

10. Rodriguez Gil FJ, Lopez Avila A. [Cholestatic hepatitis as the first manifestation of secondary syphilis]. Gastroenterol Hepatol. 2006;29(10):653.

11. Ridruejo E, Mordoh A, Herrera F, Avagnina A, Mando OO. Severe cholestatic hepatitis as the first symptom of secondary syphilis. Dig Dis Sci. 2004;49(9):1401-1404.

12. Gschwantler M, Gulz W, Schrutka-Kolbl C, Kogelbauer G, Schober G, Bibus B, Weiss W. [Acute hepatitis as the leading symptom of secondary syphilis]. Dtsch Med Wochenschr. 1996;121(47):1457-1461.

13. Gomez Rodriguez N, Formigo Rodriguez E, Ferreiro Seoane JL, Gonzalez Mediero G, Anton Badiola I. [Pol- yarthritis and hepatitis as presentation forms of secondary syphilis]. An Med Interna. 1993;10(10):492-494.

14. Balikocioglu A, Quaidoo E, Vuletin JC, Trotman BW. Hepatitis and glomerulonephritis in secondary syphilis. J Assoc Acad Minor Phys. 1991;2(2):72-75.

15. Murray FE, O'Loughlin S, Dervan P, Lennon JR, Crowe J. Granulomatous hepatitis in secondary syphilis. Ir J Med Sci. 1990;159(2):53-54.

16. Comer GM, Mukherjee S, Sachdev RK, Clain DJ. Cardiolipin-fluorescent (M1) antimitochondrial antibody and cholestatic hepatitis in secondary syphilis. Dig Dis Sci. 1989;34(8):1298-1302.

17. Williams WC, Marion GS. Secondary syphilis presenting with arthritis, hepatitis, and glucose intolerance. J Fam Pract. 1987;25(5):509-511.

18. Tosti A, Zauli D, Negosanti M, Veronesi S. [Hepatitis in secondary syphilis. Review of the literature]. G Ital Dermatol Venereol. 1983;118(4):231-234.

19. Lonshakov Iu I, Bialik LR, Ivanova SN, Petrova L, Iarakova EA. [Rare variants of secondary syphilis (hepatitis, periostitis, eye lesions)]. Vestn Dermatol Venerol. 1983;12:57-58.

20. Bermudez JR, Echevarria S, Rodriguez de Lopez C, Pons Romero F, Val Bernal F. [Acute hepatitis in secondary syphilis]. Rev Esp Enferm Apar Dig. 1983;64(6):545-548.

21. Jeanmougin M, Dive F, Civatte J. [Detection and incidence of hepatitis in secondary syphilis. Seventy casereports (author's transl)]. Sem Hop. 1982;58(4):199-203.

22. Causey JQ. Acute hepatitis of secondary syphilis. J Miss State Med Assoc. 1982;23(7):193-194.

23. Uehara Y, Horie N, Takahashi Y, Gomi T, Yuhara M, Ikegami F, Ikeda T. [A case of secondary syphilis associated with nephrotic syndrome and acute hepatitis (author's transl)]. Nihon Jinzo Gakkai Shi. 1981;23(11):14571464.

24. Guillaume JC, Lejonc JL, Touraine R. [Hepatitis of the secondary syphilis (author's transl)]. Ann Dermatol Venereol. 1981;108(3):253-256.

25. Velasco M, Gonzalez X. [Hepatitis in secondary syphilis (author's transl)]. Rev Med Chil. 1980;108(4):330-331.

26. Duffaut M, Gailemin C, Arlet P, Botreau Y, Le Tallec Y. [Hepatitis B and primo-secondary syphilis. 3 cases]. Nouv Presse Med. 1980;9(38):2845-2846.

27. Roge J, Husson JM, Biclet P, Camilleri JP, Amat D, Roge F, Bloch F, et al. [Does the hepatitis of secondary syphilis exist? Two cases (author's transl)]. Nouv Presse Med. 1979;8(41):3335-3338.

28. Bansal RC, Cohn H, Fani K, Lynfield YL. Nephrotic syndrome and granulomatous hepatitis in secondary syphilis. Arch Dermatol. 1978;114(8):1228-1229.

29. Hjort M, Olsson R, Smith U, Zettergren L. Hepatitis in secondary syphilis. Scand J Infect Dis. 1977;9(1):59-61.

30. Dyk T, Witebska B. [Case of granulomatous hepatitis in the course of acquired secondary syphilis]. Wiad Lek. 1977;30(9):711-714.

31. Congy F, Moulias R, Beaufils H, Roujeau JC, Sqalli S, Debat P, Loeper J. [Nephrotic syndrome, hepatitis and circulating anticoagulant in secondary syphilis]. Ann Med Interne (Paris). 1977;128(10):799-802. 
32. Sewell J, Ahmed MA. Letter: Secondary syphilis and hepatitis. Br Med J. 1975;3(5974):42.

33. Editorial: Secondary syphilis and hepatitis. Br Med J. 1975;1(5950):112.

34. McCracken JD, Hall WH, Pierce HI. Nephrotic syndrome and acute hepatitis in secondary syphilis. Mil Med. 1969;134(9):682-686.

35. Singh AE, Romanowski B. Syphilis: review with emphasis on clinical, epidemiologic, and some biologic features. Clin Microbiol Rev. 1999;12(2):187-209.

36. Shockman S, Buescher LS, Stone SP. Syphilis in the United States. Clin Dermatol. 2014;32(2):213-218.

37. Peterman TA, Su J, Bernstein KT, Weinstock H. Syphilis in the United States: on the rise? Expert Rev Anti Infect Ther. 2015;13(2):161-168.

38. Hunte W, al-Ghraoui F, Cohen RJ. Secondary syphi- lis and the nephrotic syndrome. J Am Soc Nephrol. 1993;3(7):1351-1355.

39. Petersen LR, Mead RH, Perlroth MG. Unusual manifestations of secondary syphilis occurring after orthotopic liver transplantation. Am J Med. 1983;75(1):166-170.

40. Agrawal NM, Sassaris M, Brooks B, Akdamar K, Hunter F. The liver in secondary syphilis. South Med J. 1982;75(9):1136-1138.

41. Larsen SA, Steiner BM, Rudolph AH. Laboratory diagnosis and interpretation of tests for syphilis. Clin Microbiol Rev. 1995;8(1):1-21.

42. Smith L, Angarone MP. Sexually Transmitted Infections. Urol Clin North Am. 2015;42(4):507-518.

43. Belum GR, Belum VR, Chaitanya Arudra SK, Reddy BS. The Jarisch-Herxheimer reaction: revisited. Travel Med Infect Dis. 2013;11(4):231-237. 